\title{
Transcatheter mitral valve-in-valve implantation using a transseptal approach
}

\author{
Zenon Huczek, Bartosz Rymuza, Piotr Scisło, Janusz Kochman, Krzysztof J. Filipiak, Grzegorz Opolski \\ $1^{\text {st }}$ Department of Cardiology, Medical University of Warsaw, Warsaw, Poland
}

Adv Interv Cardiol 2019; 15, 1 (55): 107-109

DOI: https://doi.org/10.5114/aic.2019.83775

Transcatheter mitral valve-in-valve intervention (TMVI) is an alternative mode of treatment to re-do surgery for bioprosthetic valve failure. Although transapical access represents the shortest route to deliver the new bioprosthesis in the mitral position, currently the transseptal route is becoming more popular [1, 2]. We present the first case in Poland of TMVI using transseptal access.

A 69-year-old female patient who underwent surgical mitral valve replacement with an Epic $31 \mathrm{~mm}$ (Abbot) valve, after 2 ischemic strokes with pulmonary hypertension and chronic kidney disease stage 4 (EuroSCORE II $9.8 \%$ ), developed dyspnea 3 years following surgery (NYHA class III). Echocardiographic examination revealed signs of severe stenosis and mild insufficiency of the Epic valve. After discussion at the heart team meeting, due to high risk of a re-do operation the patient was referred for TMVI.

Based on the internal lumen diameter of the Epic valve the suggested valve size should be $29 \mathrm{~mm}$ (Figure $1 \mathrm{~A}$ ), but cardiac computed tomography (CCT) revealed extensive hypodense pannus formation with mean inner lumen diameter of $21 \mathrm{~mm}$ (Figure $1 \mathrm{~B}$ ). Because of this appearance it was agreed to perform balloon sizing of the degenerated bioprosthesis with a $22 \mathrm{~mm}$ balloon catheter. The patient was screened for possible left ventricular outflow tract (LVOT) obstruction by means of neo-LVOT calculation and aorto-mitral angulation, showing no or little risk of obstruction (Figures 1 C-E) [3, 4].

The procedure was performed via the transvenous route in general anesthesia under transesophageal echocardiography (TEE) guidance. After puncture of the interatrial septum in postero-inferior location and crossing through the degenerated valve into the left ventricle, dilatation of the intra septal channel was performed with a $12 \times 40 \mathrm{~mm}$ peripheral balloon catheter. Predilation with a $22 \mathrm{~mm}$ balloon catheter was performed and upon inflation a clear waist was visible (Figure 2 A); therefore a $26 \mathrm{~mm}$ Sapien 3 (Edwards Lifesciences) valve was chosen. Despite the previous dilatation of the intra-septal channel the crossing with the delivery system showed some difficulty, but after changing to a Lunderquist Extra Stiff guidewire and some mild dilatation of the distal part of the Edwards balloon it was possible to obtain correct positioning (Figure $2 \mathrm{~B}$ ). During rapid pacing the valve was implanted in a 20\%/80\% (atrium/ventricle) depth ratio and afterwards postdilated distally with an extra $2 \mathrm{ml}$ of volume to obtain a wider, cone-shaped, ventricular end of the valve. In post-procedural angiography there were no signs of paravalvular regurgitation (Figure $2 \mathrm{C}$ ), and TEE showed good expansion of the implanted valve (Figure $2 \mathrm{D}$ ).

In carefully selected patients TMVI is a forward-looking method enabling less invasive treatment for severe mitral prosthesis failure. With the use of proper implantation technique $[5,6]$ the procedure is safe and feasible via the transfemoral venous route. The most crucial part of planning the procedure, besides screening for possible LVOT obstruction, is valve sizing. Given the possibility of late valve migration, undersizing should be avoided in most cases. In those situations the true ID should be chosen as the proper diameter to size the valve. In cases with extensive hypodense pannus formation or calcifications within the bioprosthetic valve an alternative may be used. Although controversial, balloon sizing may prove useful to determine the strength and flexibility of additional tissue found within the stent of the degenerated bioprosthesis, therefore providing new information on the appropriate transcatheter valve size for mitral valve-in-valve procedures.

\section{Acknowledgments}

The authors want to thank Prof. Markus Kassel for his technical expertise and assistance during the procedure.

Corresponding author:

Bartosz Rymuza MD, $1^{\text {st }}$ Department of Cardiology, Medical University of Warsaw, 1 a Banacha St, 02-097 Warsaw, Poland, phone: +48 505402 390, e-mail: bartosz.rymuza@gmail.com

Received: 10.10.2018, accepted: 25.12.2018. 

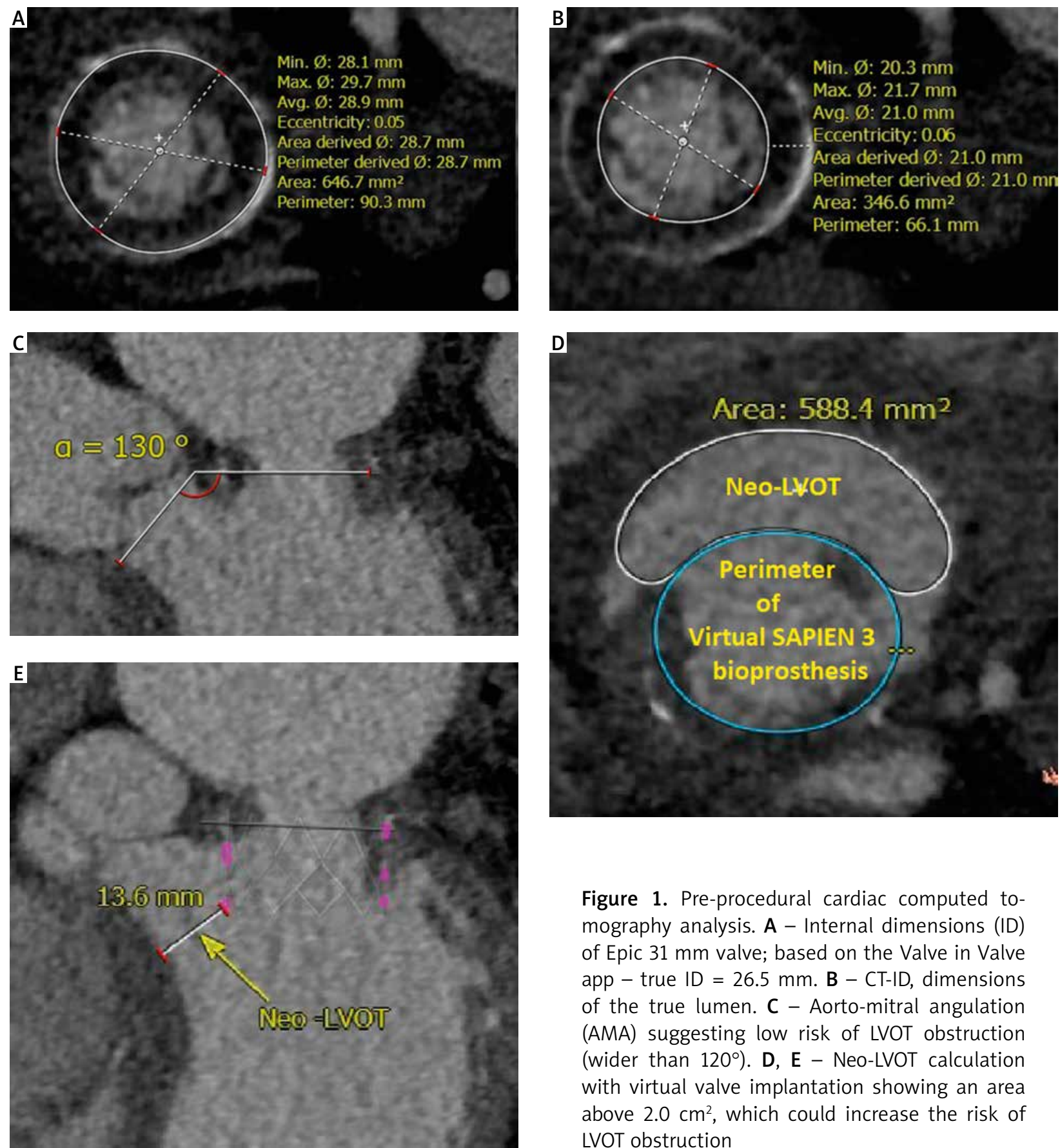

Figure 1. Pre-procedural cardiac computed tomography analysis. A - Internal dimensions (ID) of Epic $31 \mathrm{~mm}$ valve; based on the Valve in Valve app - true ID = $26.5 \mathrm{~mm}$. B - CT-ID, dimensions of the true lumen. $\mathrm{C}$ - Aorto-mitral angulation (AMA) suggesting low risk of LVOT obstruction (wider than $120^{\circ}$ ). D, E - Neo-LVOT calculation with virtual valve implantation showing an area above $2.0 \mathrm{~cm}^{2}$, which could increase the risk of LVOT obstruction 

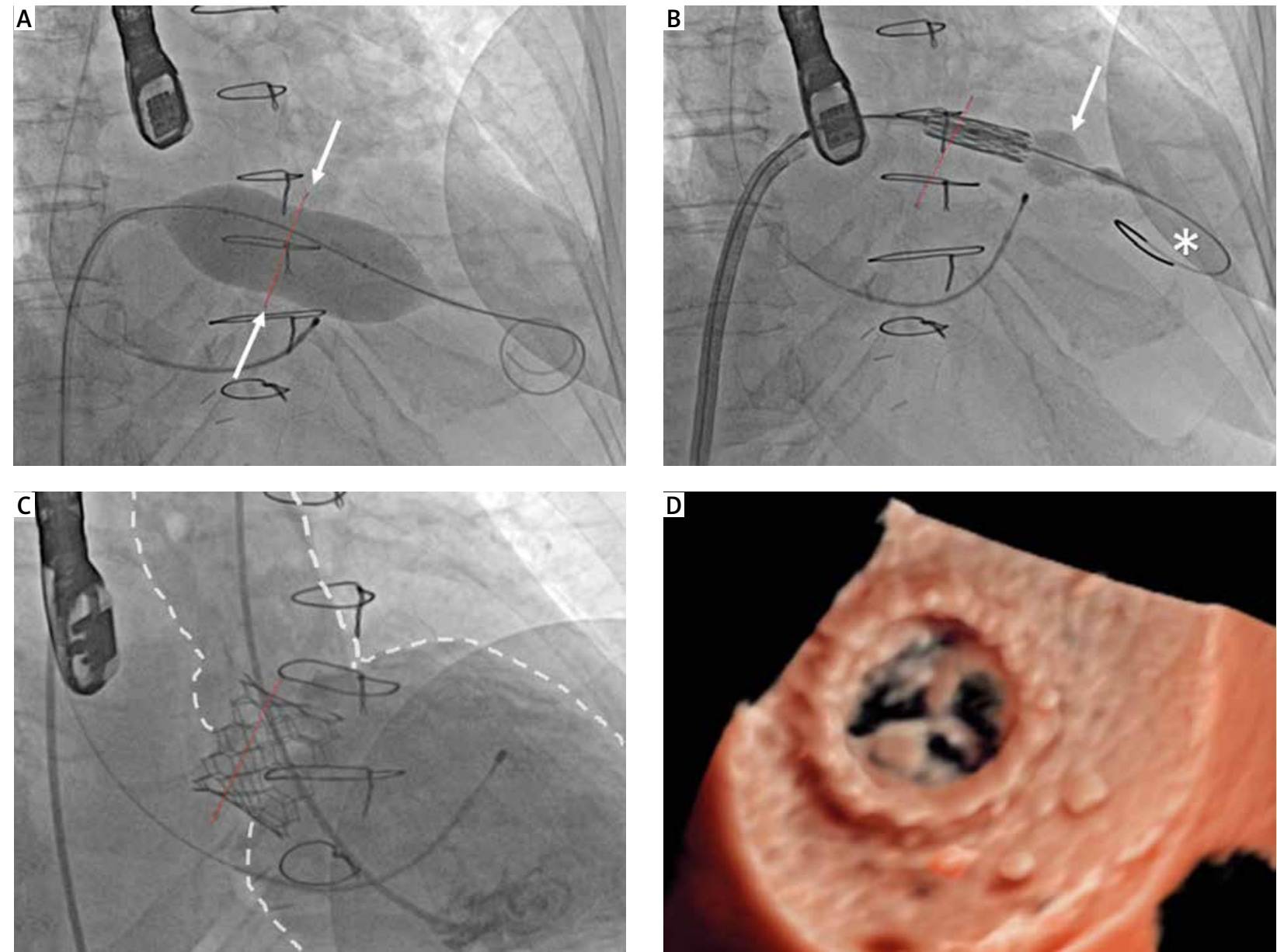

Figure 2. Transcatheter mitral valve-in-valve implantation. A - Predilatation of a $31 \mathrm{~mm}$ Epic bioprosthesis with a $22 \mathrm{~mm}$ balloon catheter. The waist is clearly visible (white arrows). B - Edwards SAPIEN $326 \mathrm{~mm}$ delivery. In order to facilitate the delivery, mild balloon inflation (white arrow) and changing to a Lunderquis Extra Stiff guidewire (asterisk) was performed. C - Final angiography showing no residual paravalvular leak (PVL). Aorta and left ventricle marked with white dotted line; red dotted line - Epic $31 \mathrm{~mm}$ valve stent. D - Postprocedural photorealistic TEE (Philips Epic CVx) showing excellent prosthesis position and no PVL

\section{Conflict of interest}

The authors declare no conflict of interest.

\section{References}

1. Kamioka N, Babaliaros V, Morse M, et al. Comparison of clinical and echocardiographic outcomes after surgical redo mitral valve replacement and transcatheter mitral valve-in-valve therapy. J Am Coll Cardiol Interv 2018; 11: 1131-8.

2. Eleid M, Whisenant B, Cabalka A, et al. Early outcomes of percutaneous transvenous transseptal transcatheter valve implantation in failed bioprosthetic mitral valves, ring annuloplasty, and severe mitral annular calcification. J Am Coll Cardiol Interv 2017; 10: 1932-42.

3. Bapat V, Pirone F, Kapetanakis S, et al. Factors influencing left ventricular outflow tract obstruction following a mitral valvein-valve or valve-in-ring procedure, part 1. Catheter Cardiovasc Interv 2015; 86: 747-60.
4. Murdoch DJ, Webb JG. LAMPOON's European vacation. Eurolntervention 2018; 14: 736-8.

5. Shivaraju A, Michel J, Frangieh A, et al. Transcatheter aortic and mitral valve-in-valve implantation using the Edwards Sapien 3 Heart Valve. J Am Heart Assoc 2018; 7: e007767.

6. Dvir D, Webb J. Mitral valve-in-valve and valve-in-ring: technical aspects and procedural outcomes. Eurolntervention 2016; 12: Y93-6. 